\title{
Tunable band gap inversion in broken gap quantum wells
}

\author{
Marcelo A. Toloza Sandoval ${ }^{1}{ }^{*}$, Tiago de Campos $^{1}$ and Guilherme M. Sipahi ${ }^{1}$ \\ 1 Instituto de Física de São Carlos, Universidade de São Paulo, Brazil. \\ *tolozasandoval@gmail.com
}

Keywords: Topological phase transition, Band hybridization, Broken gap alignment.

\section{Abstract}

In a GaSb/InAs quantum well (QW), the broken gap alignment of the energy bands enables the inversion of the fundamental gap with electrons and holes confined in different layers. In view of this peculiar feature, broken gap GaSb/InAs QWs provide a wide range of possibilities to tune the gap inversion, forming a playground for the study of the two-dimensional quantum spin Hall insulator phase, which is supported by an inverted bandstructure where the electron-hole hybridization plays a central role. In this work we consider a symmetric GaSb/InAs/GaSb QW and use the eight-band Luttinger-Kohn model to study the evolution of the fundamental gap as a function of the QW width and, in particular, the influence of an external electric field.

Without external fields, the system presents an "ordinary" behavior until the critical length of the InAs layer $(\sim 10.3 \mathrm{~nm})$, where the conduction and valence states become degenerate leading to the collapse of the fundamental band gap. The application of an external field along the growth direction breaks the spatial inversion symmetry, reopening the gap with an inversion between electron and heavy-hole bands. This inverted electron-hole bandstructure assures the existence of the two-dimensional quantum spin Hall insulator phase in a system in which this property can be externally tuned by an applied electric field.

\section{Introduction}

Heterostructures formed by the semiconductor alloys InAs and GaSb present an exotic energy band diagram where the top of the GaSb valence band is higher than the bottom of the InAs conduction band, breaking the forbidden gap at the interface, as observed in the pioneer work developed forty years ago by Sakaki et al [1]. In this so-called broken gap lineup, the band alignment allows the hybridization between the states from the InAs conduction band and the GaSb valence band, allowing the gap inversion with the electrons and holes in the same energy range, confined in the different layers and spatially separated by the interface InAs/GaSb. Over the past decades, these broken gap heterostructures have attracted much attention due to their unique properties, presenting a wide range of technological applications, as, for example, infrared detectors [2], resonant tunneling diodes [3] and spin filters [4]. Furthermore, these system are also of particular interest in fundamental physics, since electrons can move from the GaSb valence band to the InAs conduction band, leading to a semimetallic phase $[5,6]$ or even an excitonic condensate [7].
More recently, the two-dimensional quantum spin Hall phase was predicted [8] and experimentally observed $[9,10]$ in asymmetric GaSb/InAs broken gap QWs, i. e. in systems where the inverted bandstructure is present together with a strong spin-orbit coupling. In such case, the quantum confinement allows the inversion of the fundamental gap, which can be seen as a critical point separating two phases characterized by different topological numbers [11], i. e., such point defines the so-called topological phase transition in this system. Before the phase transition the system assumes an ordinary behavior and, after the gap inversion, the topological phase arises composed by the bulk insulator and metallic edges containing counterpropagating one-dimensional helical states with opposite spins, forming a two-dimensional topological insulator or quantum spin Hall system.

However, in contrast to the asymmetric case, in a symmetric GaSb/InAs broken gap QW (see Fig.1) there is a critical point (determined by the quantum confinement) where the conduction and valence states become degenerate at the center of Brillouin zone, leading to the collapse of the fundamental band gap. This is an interesting

18th Brazilian Workshop on Semiconductor Physics (BWSP 2017) 
feature since, in this case, the gap inversion occurs only by the influence of an external potential and can be exclusively controlled by an applied electric field. In particular, this setup has not been much explored in the context of the topological insulators. Therefore, further theoretical investigation of the topological phase transition, i. e., the reopening of the gap with inverted electron-hole character, in the symmetric QW case is needed.

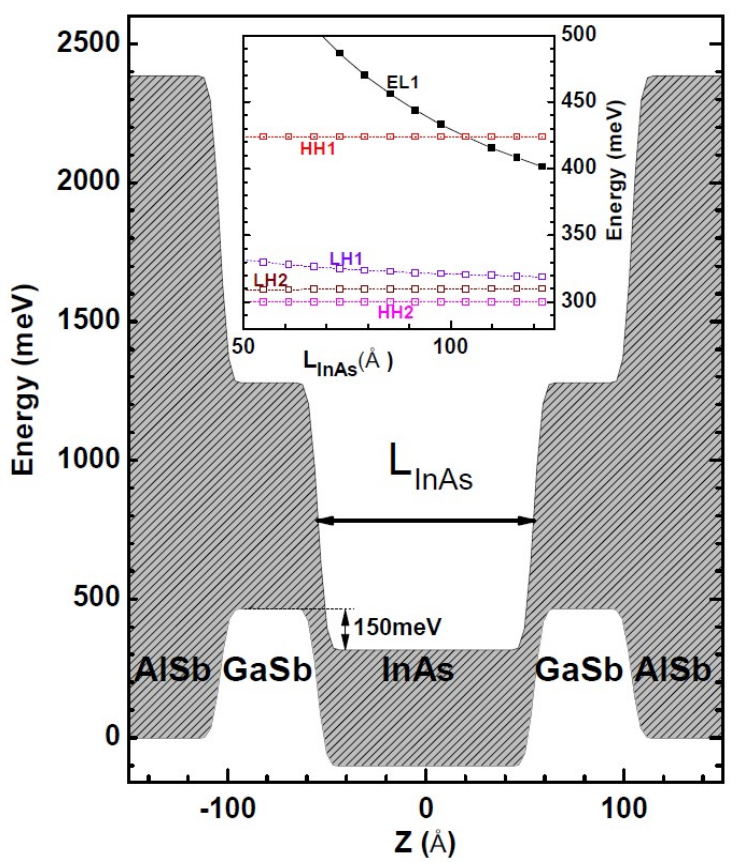

Figure 1. Energy band diagram of a symmetric AISb/GaSb/InAs/GaSb/AISb broken gap QW. The gray region stands for the forbidden gap along the growth direction of the heterostructure. Note that the top of the GaSb valence band is higher than the bottom of the InAs conduction band, forming, at the interface, a break in the gap of about 150 $\mathrm{meV}$. The inset shows the energy levels at $\mathrm{k}=0$ as a function of the InAs QW width ( $\mathrm{L}_{\ln A s}$ ), assuming a thickness of $4.8 \mathrm{~nm}$ for the GaSb layer. The bulk band parameters used were taken from Ref.[12].

\section{Methods}

To correct account for the hybridization of the conduction and valence band an accurate description of the valence band states is needed, specially the heavy- and light-hole states. The usual Kane model is not adequate for this description since it gives the wrong value for the effective mass of the heavy-hole states in certain materials [13]. In this work we used the 8-band k.p model including the conduction band state, the three valence bands, the explicit coupling between them and the Luttinger corrections for the effective masses. This model has been successfully applied in describing the electronic and spintronic properties of low dimensional semiconductor for decades $[14,15,16,17]$.

The k.p model describes a bulk material around a high symmetry point, usually the $\Gamma$-point, where the physics of interest takes place. To correctly describe it, a parameterization of the matrix elements among the basis states (Bloch functions) is derived using Group Theoretical methods. To describe a heterostructure, as a QW, we apply the envelope function approximation $[13,18]$, that defines the total wave function of a state of the system as a continuous and slowly varying function, called the envelope function, that is weighted by the Bloch's function of each material. The quantum confinement along the growth direction essentially means that we have to make the substitution $\mathrm{k}_{\mathrm{z}} \rightarrow-\mathrm{i} \partial_{\mathrm{z}}$. This approach results in a system of coupled linear differential equations. This system is solved by applying the plane wave expansion, using the Fourier transformations, and solving it from direct diagonalization methods. The parameters used in the 8-band k.p model were extracted from Ref. [12] and we used 40 planes waves which suffices to achieve energy convergence in our calculations.

In our calculations, we consider a symmetric AISb/GaSb/InAs/GaSb/AISb broken gap QW whose the energy band diagram is shown in Figure 1. The inset shows the obtained energy levels, at the $\Gamma$-point, varying the InAs QW width (LInAs) and assuming a thickness of $4.8 \mathrm{~nm}$ for the GaSb layer. In this case, the fundamental gap is given by the difference between the energies EL1 and HH1, i.e., the lowest conduction subband and the highest heavy-hole subband, respectively. Such thickness of the GaSb layer was chosen in order to push the light-hole $(\mathrm{LH})$ states outside the broken gap domain (i.e., outside the region limited by the 150 meV shown in Fig.1). As consequence, only the highest heavy-hole state remains inside this region. Note that for a narrow InAs QW, due to the strong confinement, the lowest conduction subband lies higher in energy than the highest heavy-hole band and the system behaves like an usual semiconductor. 


\section{Results and Discussion}

As seen in the inset of Figure 1, by increasing the InAs QW width the conduction subband energies decrease and the weak LH1EL1 intersubband coupling is responsible for the small variation of the LH1 level, which occurs in despite of the fixed thickness of the GaSb layer. It is interesting to observe that there is a critical thickness of the $\ln A s$ layer $\left(\operatorname{LinAs}_{\ln } \approx 9.6 \mathrm{~nm}\right)$ where the $\mathrm{EL} 1$ and $\mathrm{HH} 1$ bands become degenerate and the fundamental gap vanishes. In particular, after this critical thickness (at $\operatorname{LinAs}_{\mathrm{n}} \approx 10.4 \mathrm{~nm}$ ), the subband structure exhibits an anticrossing, at the $\Gamma$-point, between EL1 and the symmetric $\mathrm{HH} 1$ state, which results in a pair of hybridized EL$\mathrm{HH} 1$ (S) subbands at different energy levels with $\mathrm{HH}(\mathrm{A})$ state lying in between them, as in Fig. 2 (a). We label, in Figure 2, the subbands by its composition at $\Gamma$-point for the case without an applied electric field. When we apply the electric field, the state's composition and the subband ordering changes, but by looking at the general composition we can track its origin to the subbands of the symmetric case. What we observed is that the $\mathrm{HH}(\mathrm{A})$ subband is noninteracting, i. e., it remains as a $\mathrm{HH}(\mathrm{A})$ for all configurations. Furthermore, as we increase the strength of the electric field $E$, it becomes further localized at the lower GaSb barrier with decreasing energy, as seen in Figure 2(a)-(c). With the expulsion of the $\mathrm{HH}(\mathrm{A})$ band from the broken gap energy range, the subband structure starts to resemble the case of the asymmetric QW. For a field of $1 \mathrm{mv} / \mathrm{nm}$ there is already the band inversion with an overall energy gap characterizing a topological phase transition, as shown in Figure 2(b). Furthermore, due to the breaking of structural symmetry the subbands spin split, with the hole states showing a rather large spin splitting, as seen in Figure 2(b)-(d).
By further increasing the electric field to 3 $\mathrm{mV} / \mathrm{nm}$ the hybridized bands becomes well isolated from the others, specially from the $\mathrm{HH}(\mathrm{A})$ band, see Figure 2(c)-(d). Since the electric field push the hole states up and the electron states down, the anticrossing point, i. e., the $k$ point where the topological gap appears, increase in momentum. As seen in Figure 2(d), where the electric field magnitude is $5 \mathrm{mV} / \mathrm{nm}$ the anticrossing point occurs in higher momentum and the penetration of the subbands are also higher.

As we observed from these calculations, the value of the hybridized gap and the spin splitting of the subbands do not seem to increase. Rather, they seem to saturate with the increase of the electric field. This is backed by the fact that as we increase the magnitude of the electric field we are essentially recovering the asymmetric QW case. However, more detailed calculations need to be done to confirm this trend.

\section{Conclusion}

We have studied the symmetric $\mathrm{GaSb} / \mathrm{lnAs} / \mathrm{GaSb}$ QW using a 8-band k.p model under the envelope function and plane wave expansion. Using fixed GaSb layers of $4.8 \mathrm{~nm}$, we observed that, for a thickness of the InAs layer of $9.6 \mathrm{~nm}$, the fundamental gap closes with a remarkable distinction that a $\mathrm{HH}$ state remains degenerate with the EL state even varying the thickness of the InAs layer. Applying an electric field, we break the structural symmetry of the system and allow for a topological phase transition. We have shown that the subband anticrossing point and the magnitude of the topological gap can be fine tunned with the electric field. In the limit of large enough electric fields, our system resembles the asymmetric case.
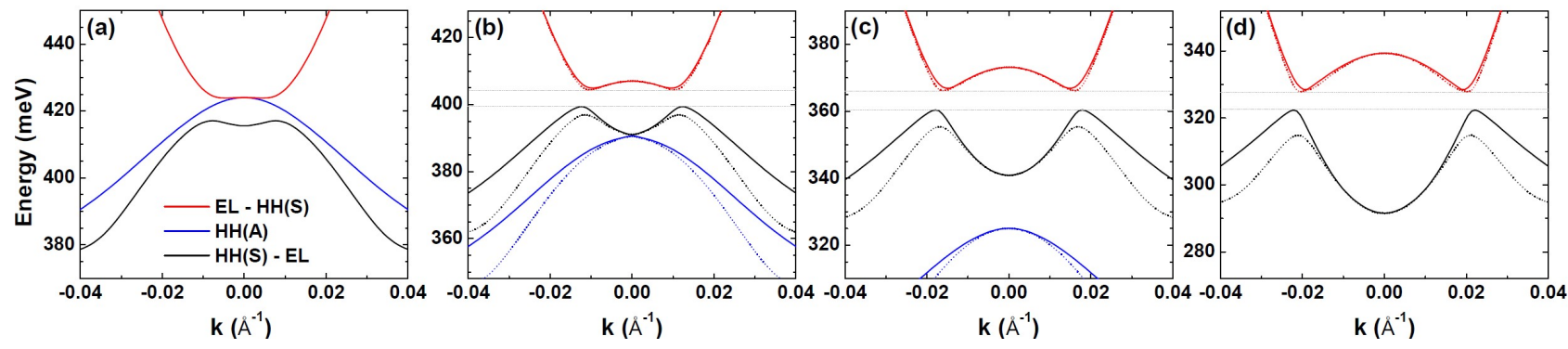

Figure 2. Band structure for a symmetric $\mathrm{AlSb} / \mathrm{GaSb} / \mathrm{InAs} / \mathrm{GaSb} / \mathrm{AlSb}$ broken gap $\mathrm{QW}$ with the fixed thicknesses $L_{\text {InAs }}=10.3 \mathrm{~nm}$ and $L_{G a S b}=4.8 \mathrm{~nm}$. (a) $E=0$, (b) $E=1 \mathrm{mV} / \mathrm{nm}$, (c) $E=3 \mathrm{mV} / \mathrm{nm}$ and (d) $E=5 \mathrm{mV} / \mathrm{nm}$. 


\section{Acknowledgments}

The authors thank the Brazilian agencies FAPESP, CNPq and CAPES for financial support.

\section{References}

[1] Sakaki, H.; Chang, L. L.; Ludeke, R.; Chang, C. A.; Sai-Halasz, G. A. and Esaki, L. Appl. Phys. Lett. 1977, 31, 211.

[2] Chow, D. H.; Miles, R. H.; Schulman, J. N.; Collins, D. A. and McGill, T. C. Semicond. Sci. Technol. 1991, 31, 115008.

[3] Ting, D. Z.-Y.; Collins, D. A.; Yu, E. T.; Chow, D. H. and McGill, T. C. Appl. Phys. Lett. 1990, 57, 1257.

[4] Ting, D. Z.-Y. and Cartoixà, X. Appl. Phys. Lett. 2002, 81, 4198 .

[5] Sai-Halasz, G. A.; Esaki, L. and Harrison, W. A. Phys. Rev. B. 1978, 18, 2812

[6] Kroemer, H. Physica E. 2004, 20, 196.

[7] Marlow, T. P.; Cooper, L. J.; Arnonr, D. D.; Patel, N. K.; Whittaker, D. M.; Linfield, E. H.; Ritchie, D. A. and Pepper, M. Phys. Rev. Lett. 1999, 82, 2362.

[8] Liu, C.; Hughes, T. L.; Qi, X.-L.; Wang, K. and Zhang, S.-C. Phys. Rev. Lett. 2008, 100, 2812.

[9] Knez, I.; Du, R.-R. and Sullivan, G. Phys. Rev. Lett. 2011, 107, 136603.

[10] Suzuki, K.; Harada, Y.; Onomitsu, K. and Muraki, K. Phys. Rev. B. 2013, 87, 235311.

[11] Qi, X.-L. and Zhang, S.-C. Rev. Mod. Phys. 2011, 83, 1057.

[12] Vurgaftman, I. and Meyer, J. R. J. Appl. Phys. 2001, 89, 5815.

[13] Bastard, G., "Wave mechanics applied to semiconductor heterostructure", Monographies de Physique, Les Editions de Physique, Les Ulis Cedex.

[14] Sipahi, G. M.; Enderlein, R.; Scolfaro, L. M. R. and Leite, J. R. Phys. Rev. B. 1996, 53, 9930.

[15] Rodrigues, S. C. P.; Scolfaro, L. M. R.; Leite, J. R. and Sipahi, G. M. Appl. Phys. Lett. 2000, 76, 1015.

[16] Rodrigues, S. C. P.; Scolfaro, L. M. R.; Leite, J. R.; da Cunha Lima, I. C.; Sipahi, G. M. and Boselli, M. A. Phys Rev. B. 2004, $70,165308$.

[17] Faria Junior, P. E. and Sipahi, G. M. J. Appl. Phys. 2012, 112, 103716

[18] Burt, M. G. J. Phys: Condens. Matter. 1999, 11, 53. 